\title{
Turbulence models of gravitational clustering
}

\author{
José Gaite \\ IDR, ETSI Aeronáuticos, Universidad Politécnica de Madrid, \\ Pza. Cardenal Cisneros 3, E-28040 Madrid, Spain \\ February 15, 2012
}

\begin{abstract}
Large-scale structure formation can be modeled as a nonlinear process that transfers energy from the largest scales to successively smaller scales until it is dissipated, in analogy with Kolmogorov's cascade model of incompressible turbulence. However, cosmic turbulence is very compressible, and vorticity plays a secondary role in it. The simplest model of cosmic turbulence is the adhesion model, which can be studied perturbatively or adapting to it Kolmogorov's non-perturbative approach to incompressible turbulence. This approach leads to observationally testable predictions, e.g., to the power-law exponent of the matter density two-point correlation function.
\end{abstract}

\section{Introduction}

The evolution of the Universe is ruled by gravity. Although the Einstein equations of General Relativity (GR) are strongly nonlinear, the early evolution of the Universe can be described by a FLRW solution plus linear perturbations. Since the FLRW solutions are unstable, perturbations grow, and they grow faster on smaller scales, becoming nonlinear and decoupling from the global expansion. As instability is an inherent property of selfgravitating systems, they are bound to cluster and collapse as long as they can dissipate their kinetic energy. This dissipation is essentially a transfer of kinetic energy from one scale to another smaller scale, due to nonlinear mode coupling. The kinetic energy eventually reaches the smallest scales and disappears (as discussed in page 31).

The nonlinear transfer of kinetic energy from larger to smaller scales is the hallmark of fluid turbulence and is the basis of Kolmogorov's cascade model of incompressible turbulence, namely, of turbulent solutions of the Navier-Stokes equations with large Reynolds number but small Mach number [1]. In cosmology and, in particular, in cold-dark-matter (CDM) models, the Mach number of the matter fluid has to be large, so the turbulence is actually very compressible. In contrast, the fluid velocities are non-relativistic (except on very small scales). Therefore, a Newtonian treatment is generally appropriate. In fact, 
the full Newtonian dynamics is still too complex for an analytical approach, so it is further simplified as follows.

\section{Zeldovich approximation and adhesion model}

The Newtonian equation of motion of a test particle in an expanding background is best expressed in terms of the comoving coordinate $\boldsymbol{x}=\boldsymbol{r} / a(t)$ and the peculiar velocity $\boldsymbol{u}=$ $\dot{\boldsymbol{x}}=\boldsymbol{v}-H \boldsymbol{r}$. So $d \boldsymbol{v} / d t=\boldsymbol{g}_{\mathrm{T}}$ is written as $d \boldsymbol{u} / d t+H \boldsymbol{u}=\boldsymbol{g}$, in terms of the peculiar gravitational field, $\boldsymbol{g}=\boldsymbol{g}_{\mathrm{T}}-\boldsymbol{g}_{\mathrm{b}}$, with background field $\boldsymbol{g}_{\mathrm{b}}=\dot{H} \boldsymbol{r}+H^{2} \boldsymbol{r}$, such that $3(\dot{H}+$ $\left.H^{2}\right)=\nabla \cdot \boldsymbol{g}_{\mathrm{b}}=-4 \pi G \rho_{\mathrm{b}}$, which is just the dynamical FLRW equation for $P=0$ (the dust model). Of course, $\boldsymbol{g}$ depends on the motion of the remaining particles, and the problem is nonlinear, like in GR. It can be linearized when the peculiar variables are small, so the motion is, approximately, $\boldsymbol{x}=\boldsymbol{x}_{0}+b(t) \boldsymbol{g}\left(\boldsymbol{x}_{0}\right)$, where $b(t)$ is the growth rate of linear density fluctuations. Redefining time as $\tau=b(t)$, the motion is simply linear motion, with a constant velocity given by the initial peculiar gravitational field. Naturally, nearby particles have different velocities, and, as the linear solution is prolonged into the nonlinear regime, trajectories cross at caustic surfaces [2], called "Zeldovich pancakes" in this context. These are supposed to be the first cosmological structures.

Actually, the simplest caustic arises from inward spherical motion (spherical collapse), which gives rise to a point singularity, that is, a zero-dimensional (degenerate) caustic. After the multiple collision of particles at one point, their evolution in Newtonian gravity is undefined. If no kinetic energy is dissipated (adiabatic collapse), the particles cross (or rebound), returning to their initial positions. This unrealistic evolution takes place at any caustic, so there is no real structure formation, unless a dissipation mechanism is introduced. Hence, the linear motion in the Zeldovich approximation is supplemented with a viscosity term, resulting in the equation

$$
\frac{d \widetilde{\boldsymbol{u}}}{d \tau} \equiv \frac{\partial \widetilde{\boldsymbol{u}}}{\partial \tau}+\widetilde{\boldsymbol{u}} \cdot \nabla \widetilde{\boldsymbol{u}}=\nu \nabla^{2} \widetilde{\boldsymbol{u}}
$$

where $\widetilde{\boldsymbol{u}}$ is the peculiar velocity in $\tau$-time. To this equation, it must be added the novorticity (potential flow) condition, $\nabla \times \widetilde{\boldsymbol{u}}=0$, implied by $\nabla \times \boldsymbol{g}\left(\boldsymbol{x}_{0}\right)=0$. Eq. (1) is the Burgers equation for very compressible (pressureless) fluids. The limit $\nu \rightarrow 0$ might seem to recover the caustic-crossing solutions but actually is the high Reynolds-number limit and gives rise to Burgers turbulence. Whereas incompressible turbulence is associated to the development of vorticity, Burgers turbulence is associated to the development of shock fronts, namely, discontinuities of the velocity. These discontinuities arise at caustics and give rise to matter accumulation by inelastic collision of particles. The viscosity $\nu$ measures the thickness of shock fronts, which become true singularities in the limit $\nu \rightarrow 0$. This is the adhesion model, which produces a characteristic network of sheets, filaments and nodes, called "the cosmic web." 


\section{Approaches to cosmic Burgers turbulence}

Unlike the Navier-Stokes equation of incompressible fluids, the Burgers equation is integrable and, therefore, keeps memory of initial conditions. On the other hand, in both incompressible and Burgers turbulence, kinetic energy cascades down to smaller scales until it is dissipated. This dissipation is of thermal nature in normal fluids; but, in cosmology, while baryons experience thermal dissipation, the "dissipation" in CDM has mainly gravitational origin [3]. Therefore, it is not sensible to just neglect the dissipated energy in Eq. (11). Moreover, the gravitational equations of motion are chaotic, like the NavierStokes equation, and tend to lose memory of initial conditions. In Burgers turbulence, we can recover the dissipated energy and have a stationary state with no memory of initial conditions (a fractal attractor, actually) by adding a "noise" to Eq. (1), giving rise to the stochastic adhesion model. A white noise is appropriate for thermal fluctuations, but we must allow for non-thermal fluctuations and also take into account the large-scale energy pumping. Both can be modeled together as some "colored" stochastic forcing. Therefore, the total random force, which must derive from a potential, $\boldsymbol{f}=\nabla \eta$, is just assumed to be Gaussian, with zero mean and white-in-time covariance:

$$
\left\langle\eta(\boldsymbol{x}, t) \eta\left(\boldsymbol{x}^{\prime}, t^{\prime}\right)\right\rangle=D\left(\boldsymbol{x}-\boldsymbol{x}^{\prime}\right) \delta\left(t-t^{\prime}\right)
$$

This stochastic adhesion model correctly describes the cosmic web structure as a "quasiVoronoi" tessellation of shock fronts [4].

Let us see, first, a perturbative approach to this model and, second, a non-perturbative one, based on the Kolmogorov approach to incompressible turbulence [5].

\subsection{Perturbative approach}

The stochastic Burgers equation has been studied in a very different context, namely, the statistical description of surface growth: when surface's height is identified with velocity potential, the KPZ equation for surface growth is equivalent to the stochastic Burgers equation [6, p. 61]. It can be studied with perturbation theory, which shows that a dynamical scaling appears at the fixed points of the dynamical renormalization group [6]. Unfortunately, the nontrivial fixed point is repulsive (in three spatial dimensions), so the nonlinear term of the Burgers equation is irrelevant (in the renormalization-group sense) and the viscous term dominates in the perturbative stationary state. Therefore, turbulence can only occur in the strong-coupling, non-perturbative regime.

In fact, the effective coupling constant in the renormalization group equations has the generic expression $\lambda^{2} D / \nu^{3}$ [6], where $\lambda$ is a coupling constant for the nonlinear term in Eq. (1), $D$ is the noise strength, as in Eq. (2), and $\nu$ is the viscosity. As turbulence occurs when $\nu \rightarrow 0$, the coupling must be strong. More precisely, the effective coupling constant is actually proportional to the cube of the Reynolds number, which has to be very large, making perturbation theory unreliable. 


\subsection{Kolmogorov like approach}

A non-perturbative approach can be based on Kolmogorov's universality assumptions, namely, statistical homogeneity, isotropy, and velocity scaling laws [1]. These laws stem from the principle that the only parameter relevant for the turbulent cascade is the energy flux across the scales, per unit time and per unit mass, $\varepsilon$. Therefore, velocity correlation functions, for example, must be power-laws, with exponents determined by dimensional analysis; in particular,

$$
\left\langle\widetilde{\boldsymbol{u}}(\boldsymbol{x}) \widetilde{\boldsymbol{u}}\left(\boldsymbol{x}^{\prime}\right)\right\rangle \propto\left(\varepsilon\left|\boldsymbol{x}-\boldsymbol{x}^{\prime}\right|\right)^{2 / 3} .
$$

From this expression and the relation $\nabla \cdot \tilde{\boldsymbol{u}} \propto \nabla \cdot \boldsymbol{g} \propto \delta \rho=\rho-\rho_{\mathrm{b}}$, we can deduce the matter-density reduced two-point correlation function:

$$
\langle\delta \rho(\boldsymbol{x}) \delta \rho(\mathbf{0})\rangle / \rho_{\mathrm{b}}^{2} \propto\langle\nabla \cdot \widetilde{\boldsymbol{u}}(\boldsymbol{x}) \nabla \cdot \widetilde{\boldsymbol{u}}(\mathbf{0})\rangle \propto|\boldsymbol{x}|^{-4 / 3} .
$$

In fact, the preceding relation between $\widetilde{\boldsymbol{u}}$ and $\rho$ is only approximate and, furthermore, one should take account of intermittency, which causes deviations from Kolmogorov's scaling laws [1. Intermittency is due to spatial variations of $\varepsilon$, which are especially strong in Burgers turbulence, because dissipation takes place in caustics. A detailed analysis [5] shows that Eq. (3) holds, but the power-law exponent absolute value can be in the range $(1,4 / 3)$, depending on the noise strength. These values are smaller than the observational value, about 1.7 [7].

\section{Discussion}

The derivation of the adhesion model involves drastic simplifications, and one could object to Eq. (1) that it has almost lost track of gravity, which only survives in the definition of $\tau$. However, the essential feature of the adhesion model is that it gives rise to caustics, and this is a general feature of the irrotational motion of pressureless matter in Newtonian dynamics or in GR (the significance and genericity of caustic singularities in GR are discussed by, e.g., Landau and Lifshitz [8]). Since GR is nonlinear, just the definition of sheet, filament, and node singularities demands a special study 9. Nodes, as point singularities, are the strongest singularities and must give rise to black holes. A black hole has (quantum) internal configurations and entropy, which reflect the energy dissipated in its formation. Moreover, black holes have negative specific heat (like Newtonian self-gravitating systems) and cannot reach thermal equilibrium in an unbounded environment [10], so they have to keep growing. Black-hole growth involves a "mixmaster" dynamics that exemplifies the "gravitational turbulence" of general solutions of the Einstein equations [11. The formation and growth of super-massive black holes are probably the most dissipative processes in astrophysics and surely have contributed greatly to shape our present universe.

In any case, the adhesion model, in which all these small-scale processes are lumped into an effective viscosity, seems to be adequate to describe the formation of large-scale structure, at least, in an early stage and semiquantitatively. The construction of more realistic models of cosmic turbulence, whether Newtonian or relativistic, demands further theoretical developments. 


\section{References}

[1] U. Frisch, Turbulence: The Legacy of A.N. Kolmogorov, Cambridge University Press, 1995.

[2] S. F. Shandarin, and Ya. B. Zeldovich, Rev. Mod. Phys. 61, 185-220 (1989).

[3] A. V. Gurevich, and K. P. Zybin, Sov. Phys. Usp. 38, 687-722 (1995).

[4] S. A. Molchanov, D. Surgailis, and W. A. Woyczynski, Ann. App. Probab. 7, 200-228 (1997).

[5] J. Gaite, "Turbulence model of the cosmic structure," arXiv:1202.3011.

[6] A.-L. Barabasi, and H. E. Stanley, Fractal Concepts in Surface Growth, Cambridge University Press, 1995.

[7] B. J. Jones, V. J. Martínez, E. Saar, and V. Trimble, Rev. Mod. Phys. 76, 1211 (2004).

[8] L. D. Landau, and E. M. Lifshitz, The Classical Theory of Fields, Pergamon Press, Oxford, 1975.

[9] R. Geroch, and J. Traschen, Phys. Rev. D 36, 1017-31 (1987).

[10] S. W. Hawking, Phys. Rev. D 13, 191-197 (1976).

[11] J. D. Barrow, Phys. Rep. 85, 1-49 (1982). 